\title{
Spectrum for non-magnetic Mott insulators from power functional within Reduced Density Matrix Functional Theory
}

\author{
Y. Shinohara ${ }^{1}$, S. Sharma ${ }^{1,2}$ * S. Shallcross ${ }^{3}$, J. K. Dewhurst ${ }^{1}$, N. N. Lathiotakis ${ }^{1,4}$, and E. K. U. Gross ${ }^{1}$ \\ 1 Max-Planck-Institut für Mikrostrukturphysik, Weinberg 2, D-06120 Halle, Germany. \\ 2 Department of Physics, Indian Institute of Technology, Roorkee, 247667, Uttarkhand, India. \\ 3 Lehrstuhl für Theoretische Festkörperphysik, Staudstr. 7-B2, 91058 Erlangen, Germany. and \\ 4 Theoretical and Physical Chemistry Institute, National Hellenic Research Foundation, \\ Vass. Constantinou 48, GR-11635 Athens, Greece
}

(Dated: October 26, 2018)

\begin{abstract}
A fully first principles theory capable of treating strongly correlated solids remains the outstanding challenge of modern day materials science. This is exemplified by the transition metal oxides, prototypical Mott insulators, that remain insulating even in the absence of long range magnetic order. Capturing the non-magnetic insulating state of these materials presents a difficult challenge for any modern electronic structure theory. In this paper we demonstrate that reduced density matrix functional theory, in conjunction with the power functional, can successfully treat the nonmagnetic insulating state of the transition metal oxides $\mathrm{NiO}$ and $\mathrm{MnO}$. We show that the electronic spectrum retains a gap even in the absence of spin order. We further discuss the detailed way in which RDMFT performs for Mott insulators and band insulators, finding that for the latter occupation number minimization alone is required, but for the former full minimization over both occupation numbers and natural orbitals is essential.
\end{abstract}

PACS numbers: 71.10.-w, 71.27.+a, 71.45.Gm, 71.20.Nr

\section{INTRODUCTION}

One of the most useful derivatives of a groundstate density functional theory (DFT) calculation is the Kohn-Sham (KS) eigenvalues[1], which lead to a non-interacting spectrum. Even though the KS equations represent an auxiliary non-interacting system whose states and eigenvalues may be quite different from the true quasi-particle system, empirical evidence shows that in many cases this single particle KS spectrum is in agreement with the X-ray photo-emission Spectroscopy (XPS) and Bremsstrahlung isochromat spectroscopy (BIS) experiments 22 5. However, for strongly correlated materials this KS spectrum is found to be in fundamental disagreement with experimental reality. In the absence of spin-ordering all modern exchange correlation (xc) functionals within DFT fail to predict an insulating ground-state for transition metal mono-oxides (TMOs), the prototypical Mott insulators. On the other hand, it is well known experimentally that these materials are insulating in nature even at elevated temperatures (much above the Néel temperature) 6, 7]. This indicates that magnetic order is not the driving mechanism for the gap, but merely a co-occurring phenomenon. In fact not only DFT, but most modern many-body techniques such as the $G W$ method also fail to capture the insulating behavior in TMOs without explicit long range spin ordering [8-10].

In this regard, the two many-body techniques that are able to capture the correct physics of strong correlations are dynamical mean field theory (DMFT) [11-13] and reduced density matrix functional theory (RDMFT) [14; these two methods predicts TMOs as insulators, even in the absence of long range spin-order. This clearly points towards the ability of these techniques to capture physics well beyond the reach of most modern day ground-state methods.

Despite this success the effectiveness of RDMFT as a ground state theory has been seriously hampered due to the absence of a technique for the determination of the spectral information. Recently, this final hurdle has also been removed and the spectral information thus obtained for TMOs was shown to be in good agreement with experiments [15. However, these spectra were calculated in the presence of anti-ferromagnetic order. The question then arises as to how effective RDMFT is in describing the insulating state of Mott insulators in the absence of long range spin order. In order to answer this question, in the present work, we study the spectral properties of non-magnetic $\mathrm{NiO}$ and $\mathrm{MnO}$. Here former is insulating due to interplay of Mott localization and charge transfer effects while the latter is insulating purely due to strong Mott localization. A detailed analysis of RDMFT and KS orbitals is performed which shows that, unlike in the case of band insulators, for Mott insulators the nature of two set of orbitals are very different and this difference is indeed crucial for the success of RDMFT in describing Mott physics. 


\section{THEORY}

Within RDMFT the one-body reduced density matrix (1-RDM) is the basic variable [16, 17]

$\gamma\left(\mathbf{r}, \mathbf{r}^{\prime}\right)=N \int d \mathbf{r}_{2} \ldots d \mathbf{r}_{N} \Phi^{*}\left(\mathbf{r}^{\prime}, \mathbf{r}_{2} \ldots \mathbf{r}_{N}\right) \Phi\left(\mathbf{r}, \mathbf{r}_{2} \ldots \mathbf{r}_{N}\right)$

where $\Phi$ denotes the many-body wave function. Diagonalization of this matrix produces a set of natural orbitals [16], $\phi_{j \mathbf{k}}$, and occupation numbers, $n_{j \mathbf{k}}$, leading to the spectral representation

$$
\gamma\left(\mathbf{r}, \mathbf{r}^{\prime}\right)=\sum_{j, \mathbf{k}} n_{j \mathbf{k}} \phi_{j \mathbf{k}}(\mathbf{r}) \phi_{j \mathbf{k}}^{*}\left(\mathbf{r}^{\prime}\right)
$$

where the necessary and sufficient conditions for ensemble $N$-representability of $\gamma\left[18\right.$ require $0 \leq n_{j \mathbf{k}} \leq 1$ for all $j, \mathbf{k}$, and $\sum_{j, \mathbf{k}} n_{j \mathbf{k}}=N$. Here $j$ represents the band index and $\mathbf{k}$ the crystal momentum.

In terms of $\gamma$, the total ground state energy [17] of the interacting system is (atomic units are used throughout)

$$
\begin{aligned}
E[\gamma]= & -\frac{1}{2} \int \lim _{\mathbf{r} \rightarrow \mathbf{r}^{\prime}} \nabla_{\mathbf{r}}^{2} \gamma\left(\mathbf{r}, \mathbf{r}^{\prime}\right) d^{3} r^{\prime}+\int \rho(\mathbf{r}) V_{\mathrm{ext}}(\mathbf{r}) d^{3} r \\
& +\frac{1}{2} \int \frac{\rho(\mathbf{r}) \rho\left(\mathbf{r}^{\prime}\right)}{\left|\mathbf{r}-\mathbf{r}^{\prime}\right|} d^{3} r d^{3} r^{\prime}+E_{\mathrm{xc}}[\gamma]
\end{aligned}
$$

where $\rho(\mathbf{r})=\gamma(\mathbf{r}, \mathbf{r}), V_{\text {ext }}$ is a given external potential, and $E_{\mathrm{xc}}$ we call the xc energy functional. In principle, Gilbert's 17] generalization of the Hohenberg-Kohn theorem to the 1-RDM guarantees the existence of a functional $E[\gamma]$ whose minimum yields the exact $\gamma$ and the exact ground-state energy of systems characterized by the external potential $V_{\text {ext }}(\mathbf{r})$. In practice, however, the correlation energy is an unknown functional of the 1-RDM and must be approximated. Although there are several known approximations for the xc energy functional[1934, the most promising for extended systems is the power functional [14, 15] where the xc energy reads

$E_{\mathrm{xc}}[\gamma]=E_{\mathrm{xc}}\left[\left\{\phi_{i \mathbf{k}}\right\},\left\{n_{i \mathbf{k}}\right\}\right]=-\frac{1}{2} \iint d^{3} r^{\prime} d^{3} r \frac{\left|\gamma^{\alpha}\left(\mathbf{r}, \mathbf{r}^{\prime}\right)\right|^{2}}{\left|\mathbf{r}-\mathbf{r}^{\prime}\right|}$

here $\gamma^{\alpha}$ indicates the power used in the operator sense i.e.

$$
\gamma^{\alpha}\left(\mathbf{r}, \mathbf{r}^{\prime}\right)=\sum_{i} n_{i}^{\alpha} \phi_{i}(\mathbf{r}) \phi_{i}^{*}\left(\mathbf{r}^{\prime}\right)
$$

for $\alpha=1 / 2$ this is the Müller functional 35, which is known to severely overestimate electron correlation [3639 while for $\alpha=1$ this functional is equivalent to the Hartree-Fock method, which includes no correlations. If $\alpha$ is chosen to be $1 / 2<\alpha<1$, the power functional interpolates between the uncorrelated Hartree-Fock limit and the over-correlating Müller functional.
All calculations are performed using the full-potential linearized augmented plane wave code Elk 40, with practical details of the calculations following the schemes described in Refs. 14 and 15 .

\section{RESULTS}

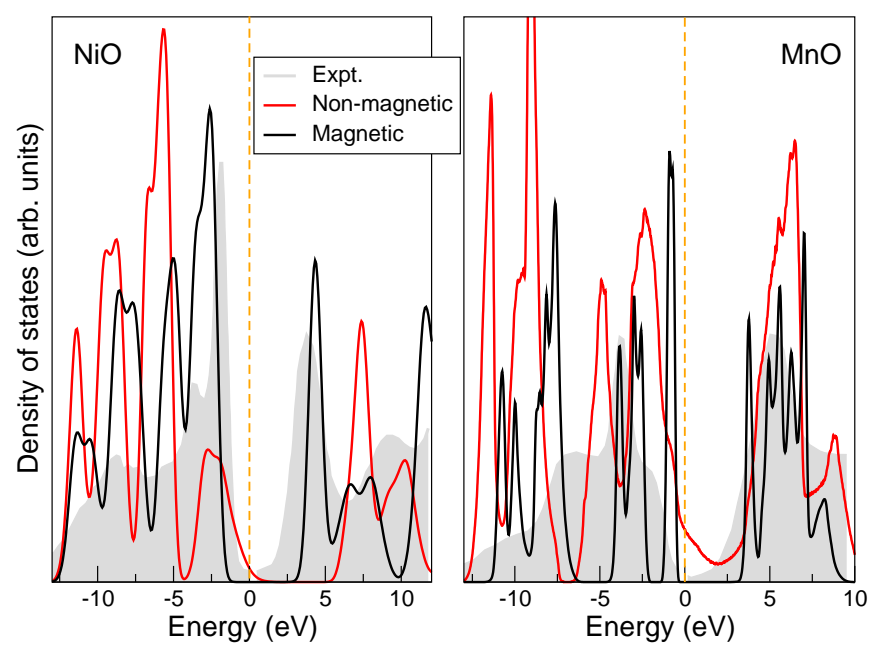

FIG. 1: (color online) Density of states as a function of energy (in eV) for $\mathrm{NiO}$ (left panel) and $\mathrm{MnO}$ (right panel). Results are obtained with (black) and without (red) long range (antiferromagnetic) spin order. For comparison experimental data taken from Refs. 3 and 5 is also shown (grey shaded area). Chemical potential is shown at dotted vertical line

Presented in Fig. 1 are the spectra for the Mott insulators under consideration. It is immediately apparent that RDMFT captures the essence of Mott-Hubbard physics: both $\mathrm{NiO}$ and $\mathrm{MnO}$ present substantial gaps at the Fermi energy and are thus insulating in the absence of spin order. This fact was already noticed in a previous work 14 in which the presence of gap without any spin-order was deduced via very different technique, namely the discontinuity in the chemical potential as a function of the particle number. A comparison of the non-magnetic spectra with the experimental data shows that the shape of the conduction band is well reproduced for both materials, but that the shape of the valence band is not in very good agreement with experiments. This agreement improves on inclusion of the spin order, indicating that even though insulating nature of TMO's is not driven by spin order, spin polarization significantly effects the spectra of these materials. This is hardly surprising given that $\mathrm{NiO}$ and $\mathrm{MnO}$ have very large local moments of $1.9 \mu_{B}$ and $4.7 \mu_{B}$ respectively.

Correct treatment of correlations is crucial for TMOs, the prototypical strongly correlated materials. As mentioned above the power functional interpolates between two limits - the highly over correlated Müller $(\alpha=0.5)$ and totally uncorrelated Hartree-Fock $(\alpha=1)$. We now 


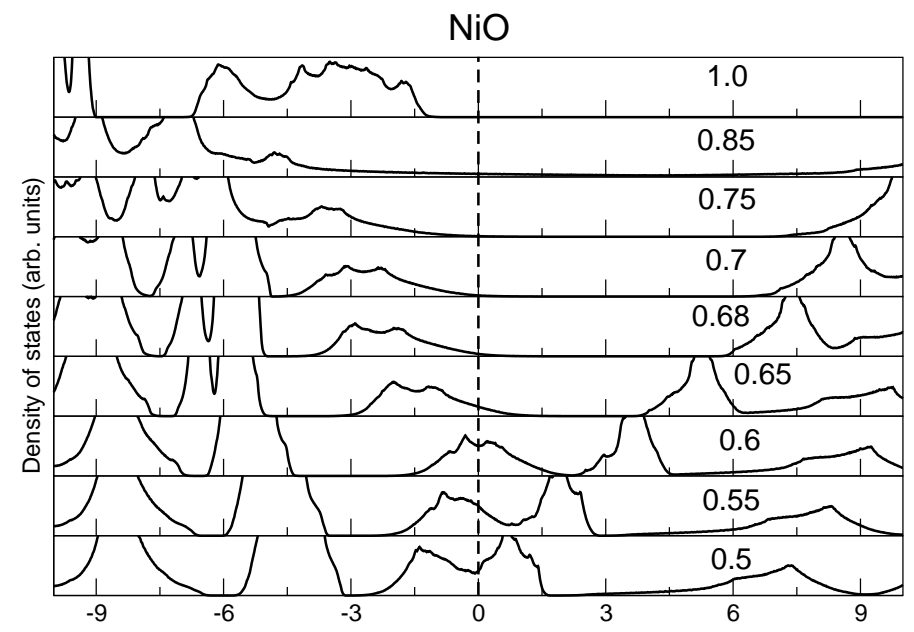

$\mathrm{MnO}$

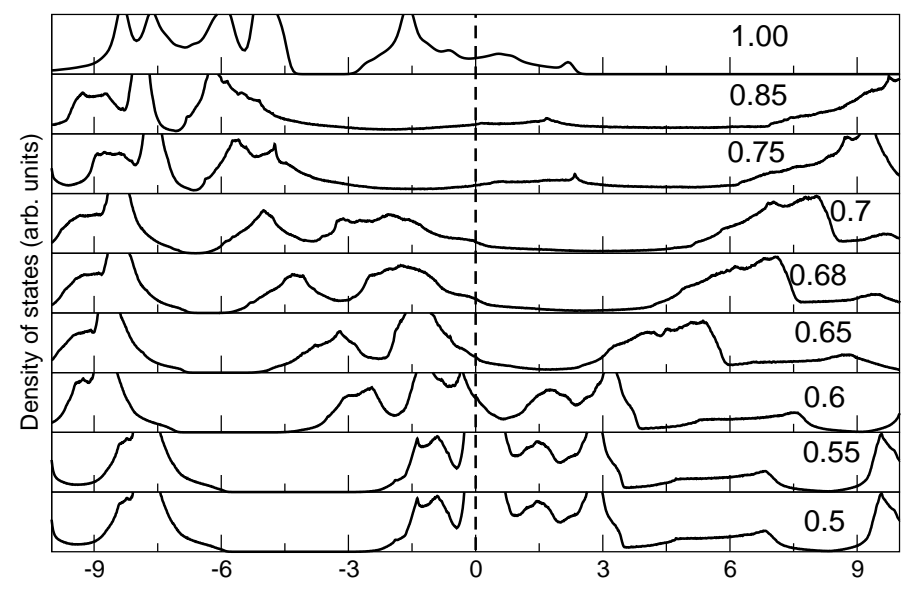

Si

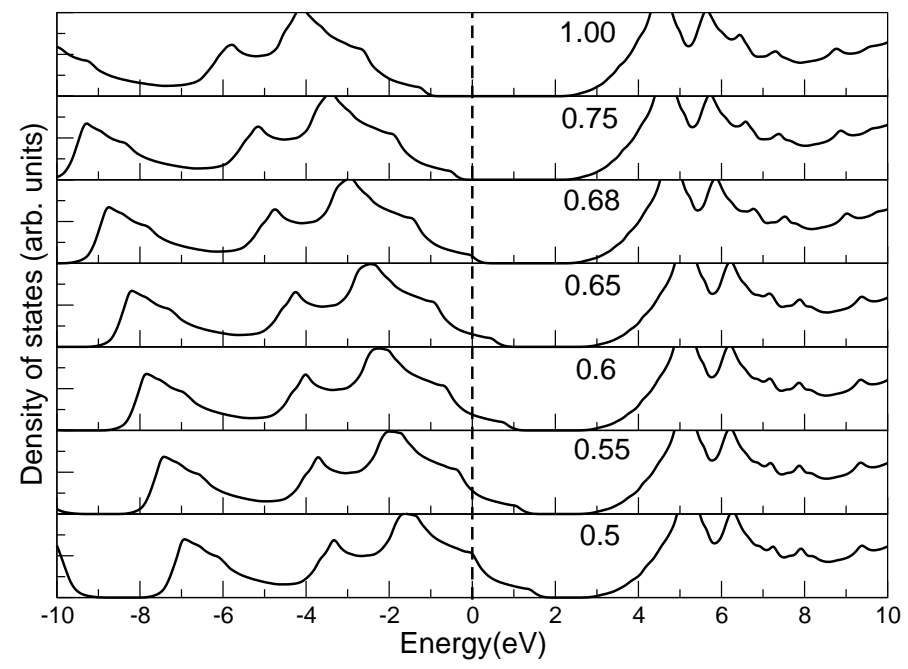

FIG. 2: Density of states as a function of energy (in $\mathrm{eV}$ ) for $\mathrm{NiO}$ (upper panels), $\mathrm{MnO}$ (middle panels), and $\mathrm{Si}$ (lower panels). The results are obtained using different values of $\alpha$ in Eq. 4 look at the effect of correlations, by varying $\alpha$, on the spectra of Mott insulators $(\mathrm{NiO}$ and $\mathrm{MnO}$ ) and band insulator (Si), see Fig. 22 The behaviour of the spectra as a function of $\alpha$ is rather trivial for band insulator, $\mathrm{Si}$; the valence bands rigidly shift lower in energy leading to increase in the band gap. The behaviour for Mott insulators is different in that the shape of the bands change as a function of $\alpha$. Both for $\mathrm{NiO}$ and $\mathrm{MnO}$ over correlated Müller functional incorrectly gives a metallic ground-state. For $\mathrm{NiO}$, which has even number of electrons in a unit-cell, the Hartree-Fock method leads to a very large band gap insulator. In contrast to this, for $\mathrm{MnO}$, with odd number of electrons in the unit-cell, a single particle theory such as Hartree-Fock can only give rise to a metallic ground state. This leads to highly non trivial behaviour for $\mathrm{MnO}$ as a function of $\alpha$, which must lie within a small range (between 0.65 and 0.7 ) in which the correct insulating ground-state is obtained. Reassuringly, this is also the range of $\alpha$ in which correct ground state behaviour is seen for $\mathrm{NiO}$.

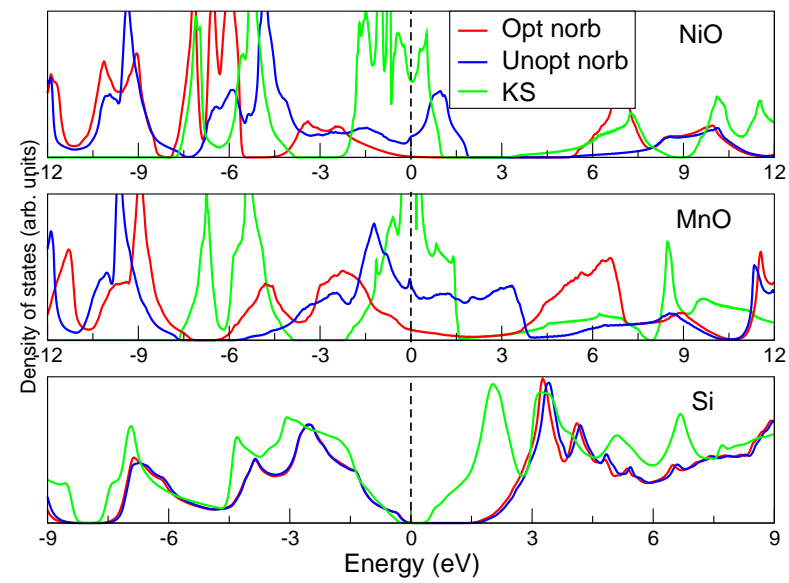

FIG. 3: Density of states as a function of energy (in eV) for $\mathrm{NiO}$ (top panel), $\mathrm{MnO}$ (middle panel) and $\mathrm{Si}$ (lower panel). Results are obtained with (black) and without (red) optimization of the natural orbitals with in RDMFT. KS results (green) are obtained using local density approximation 41.

Within RDMFT there are no Kohn-Sham-like equations to solve, and a direct minimization over natural orbitals and occupation numbers is required while maintaining the ensemble $N$-representability conditions. The minimization over occupation numbers is computationally very efficient (for details see Ref. 14), but the same cannot be said about the minimization over the natural orbitals. In practical terms, the natural orbitals (see Eq. (2)) are expanded in a set of previously converged KS states, and optimization of the natural orbitals is performed by varying the expansion coefficients. This procedure allows us to examine how different KS states are from fully optimized natural orbitals. In the present work these KS states were obtained using local density approximation (LDA) 41]. 
In Fig. 3 three set of results are shown; (i) KS density of states, (ii) RDMFT density of states obtained without optimizing the natural orbitals i.e. by using KS orbitals as natural orbitals but fully optimizing the occupation numbers and (iii) the fully optimized RDMFT results i.e. full optimization over the natural orbitals and occupation numbers. From these results it is clear that for the band insulator $\mathrm{Si}$ it is sufficient to optimize the occupation numbers to increase the band gap in line with experiment; the KS states are evidently already a very good representation of the natural orbitals. These results are in line with our experience with finite systems which shows that orbital optimization results roughly up to $25 \%$ of the total correlation energy and the rest $75 \%$ comes from the occupation numbers optimization. As may be seen in Fig. 3 the opposite situation holds for the case of the Mott insulators $\mathrm{NiO}$ and $\mathrm{MnO}$ : clearly the $\mathrm{KS}$ states differ profoundly from the natural orbitals. In this case it is crucial to optimize the natural orbitals. The reason for this is that in the case of Mott insulators it is the localization of electrons which leads to formation of the gap and KS orbitals are not sufficiently localized, thus optimization over the natural orbitals is required.

A confirmation of this charge localization may be seen in the charge density. In Fig. 4 we plot the difference $\rho(\mathbf{r})-\rho_{L D A}(\mathbf{r})$, for (i) RDMFT (lower panel) and (ii) the LSDA $+U$ functonal[42] (upper panel) within DFT for NiO. LSDA $+U$ method is chosen because, like RDMFT, it also finds the correct insulating ground state for $\mathrm{NiO}[8,43$. The LSDA $+U$ method achieves this by both spin order an on-site Hubbard $U$ and, in contrast to RDMFT, cannot treat the non-magnetic insulating state of this material. The impact of this difference on the charge density is clear in Fig. 45 significant charge localization is seen only in the RDMFT density. Interestingly, one observes an almost spherical charge accumulation at the oxygen site, a result in agreement with experiment 44, but different from that found in the corresponding $\mathrm{LSDA}+U$ result.

\section{SUMMARY}

To summarize, in this work we demonstrate that RDMFT in conjunction with the power functional is able to capture the insulating state of $\mathrm{NiO}$ and $\mathrm{MnO}$ in absence of long range spin order. However, while spin order does not drive the insulating ground state, the large local moments in these materials require spin be explicitly taken into account for excellent agreement with experimental spectra to be obtained. The power, $\alpha$, in the power-functional is an indicator of the amount of correlation and a detailed analysis shows a highly non trivial behaviour of the spectra, for Mott insulators, as a function of $\alpha$, which must lie within a small range (between 0.65 and 0.7 ) for the correct insulating ground-state is
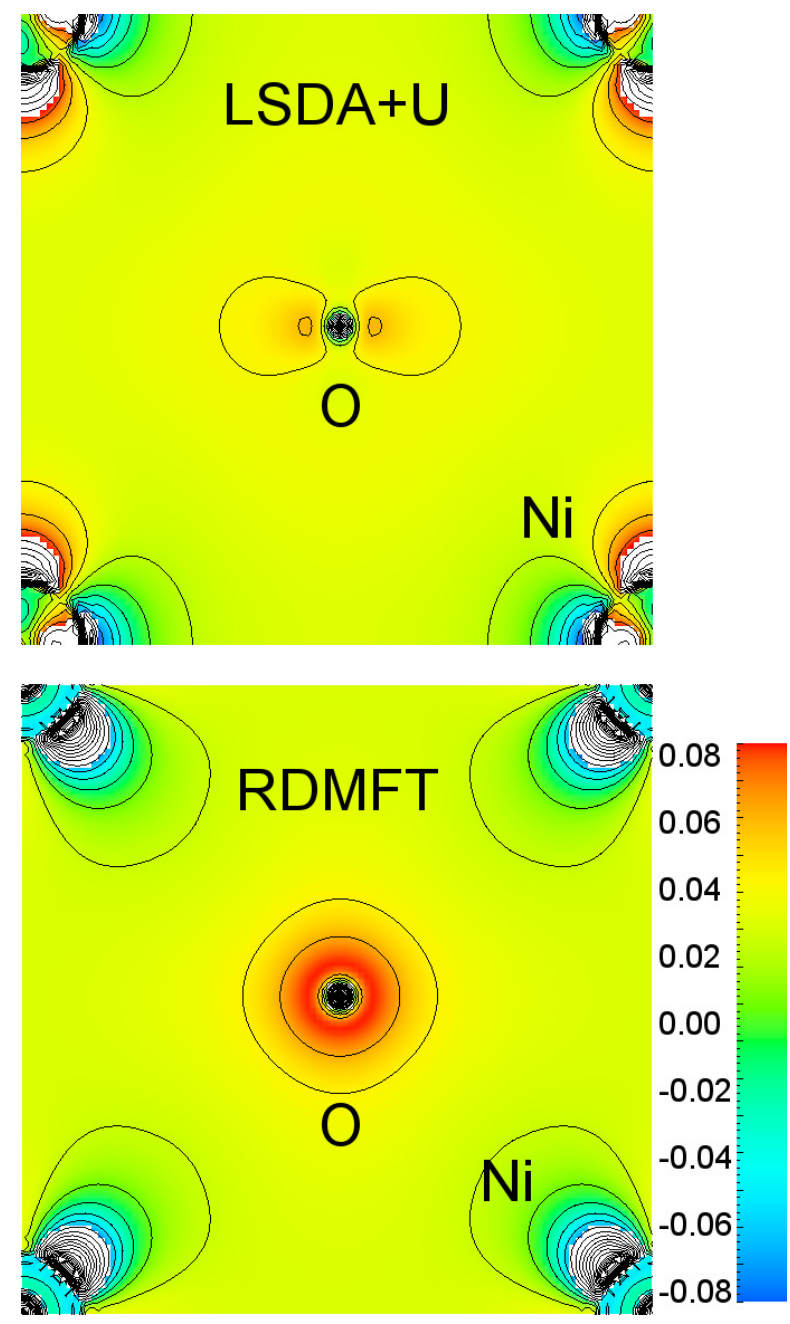

FIG. 4: (Color online) Difference between the LSDA charge density and charge density calculated using LSDA $+U$ and RDMFT, $\left(\rho(\mathbf{r})-\rho_{L S D A}(\mathbf{r})\right)$ for $\mathrm{NiO}$. Positive values indicate localization of charge as compared to LSDA.

obtained. It is further shown that the natural orbitals for the strongly correlated materials, $\mathrm{NiO}$ and $\mathrm{MnO}$, are much more localized as compared to the Kohn-Sham orbitals, which enables them to capture the physics of Mott localization in these materials.

* Electronic address: sharma@mpi-halle.mpg.de

[1] W. Kohn and L. J. Sham, Phys. Rev. 140, A1133 (1965).

[2] K. Ulmer, Phys. Rev. Lett. 3, 514 (1959).

[3] J. van Elp, R. H. Potze, H. Eskes, R. Berger, and G. A. Sawatzky, Phys. Rev. B 44, 1530 (1991).

[4] J. van Elp, J. L. Wieland, H. Eskes, P. Kuiper, G. A. Sawatzky, F. M. F. de Groot, and T. S. Turner, Phys. Rev. B 44, 6090 (1991).

[5] G. A. Sawatzky and J. W. Allen, Phys. Rev. Lett. 53, 2339 (1984).

[6] O. Tjernberg et al., Phys. Rev. B 54, 10245 (1996). 
[7] W. Jauch and M. Reehuis, Phys. Rev. B 70, 195121 (2004).

[8] C. Rödl, F. Fuchs, J. Furthmüller, and F. Bechstedt, Phys. Rev. B 79, 235114 (2009).

[9] F. Aryasetiawan and O. Gunnarsson, Phys. Rev. Lett. 74, 3221 (1995).

[10] S. Kobayashi, Y. Ohara, S. Yamamoto, and T. Fujiwara, Phys. Rev. B 78, 155112 (2008).

[11] X. Ren, I. Leonov, G. Keller, M. Kollar, I. Nekrasov, and D. Vollhardt, Phys. Rev. B 74, 195114 (2006).

[12] J. Kunes, A. V. Lukoyanov, V. I. Anisimov, R. T. Scalettar, and W. E. Pickett, Nat. Mat. 7, 198 (2008).

[13] O. Miura and T. Fujiwara, Phys. Rev. B 77, 195124 (2008).

[14] S. Sharma, J. K. Dewhurst, N. N. Lathiotakis, and E. K. U. Gross, Phys. Rev. B 78, 201103 (2008).

[15] S. Sharma, J. K. Dewhurst, S. Shallcross, and E. K. U. Gross, Phys. Rev. Lett. 110, 116403 (2013).

[16] P. O. Lödwin, Phys. Rev. 97, 1974 (1955).

[17] T. L. Gilbert, Phys. Rev. B 12, 2111 (1975).

[18] A. Coleman, Rev. Mod. Phys. 35, 668 (1963).

[19] A. M. K. Müller, Phys. Rev. A 105, 446 (1984).

[20] O. Gritsenko, K. Pernal, and E. J. Baerends, J. Chem. Phys. 122, 204102 (2005).

[21] D. R. Rohr, J. Toulouse, and K. Pernal, Phys. Rev. A 82, 052502 (2010).

[22] D. R. Rohr, K. Pernal, O. V. Gritsenko, and E. J. Baerends, J. Chem. Phys. 129, 164105 (2008).

[23] M. A. L. Marques and N. N. Lathiotakis, Phys. Rev. A 77, 032509 (2008).

[24] M. Piris, Int. J. Quant. Chem 106, 1093 (2006).

[25] K. J. H. Giesbertz, O. V. Gritsenko, and E. J. Baerends, Phys. Rev. Lett. 105, 013002 (2010).

[26] M. Piris, X. Lopez, F. Ruipérez, J. M. Matxain, and J. M. Ugalde, J. Chem. Phys. 134, 164102 (2011).
[27] K. Pernal, J. Chem. Theory Comput. 10, 4332 (2014).

[28] N. N. Lathiotakis, N. Helbig, A. Rubio, and N. I. Gidopoulos, Phys. Rev. A 90, 032511 (2014).

[29] M. Piris, J. M. Matxain, and X. Lopez, J. Chem. Phys. 139, 234109 (2013).

[30] K. J. H. Giesbertz, K. Pernal, O. V. Gritsenko, and E. J. Baerends, J. Chem. Phys. 130, 114104 (2009).

[31] M. Piris, J. M. Matxain, X. Lopez, and J. M. Ugalde, J. Chem. Phys. 136, 174116 (2012).

[32] K. Chatterjee and K. Pernal, J. Chem. Phys. 137, 204109 (2012).

[33] R. van Meer, O. V. Gritsenko, K. J. H. Giesbertz, and E. J. Baerends, J. Chem. Phys. 138, 094114 (2013).

[34] N. N. Lathiotakis, N. Helbig, A. Rubio, and N. I. Gidopoulos, J. Chem. Phys. 141, 164120 (2014).

[35] A. M. K. Müller, Phys. Lett. 105A, 446 (1984).

[36] G. Csányi and T. A. Arias, Phys. Rev. B 61, 7348 (2000).

[37] O. Gritsenko, K. Pernal, and E. J. Baerends, J. Chem. Phys. 122, 204102 (2005).

[38] J. M. Herbert and J. E. Harriman, Chem. Phys. Lett. 382, 142 (2003).

[39] N. N. Lathiotakis, N. Helbig, and E. K. U. Gross, Phys. Rev. B 75, 195120 (2007).

[40] (2004), URL http://elk.sourceforge.net

[41] J. P. Perdew and Y. Wang, Phys. Rev. B 45, 13244 (1992).

[42] A. I. Liechtenstein, V. I. Anisimov, and J. Zaanen, Phys. Rev. B 52, R5467 (1995).

[43] Y. Shinohara, S. Sharma, J. K. Dewhurst, S. Shallcross, N. N. Lathiotakis, and E. K. U. Gross, URL http:// arxiv.org/abs/1206.1713.

[44] S. L. Dudarev, L.-M. Peng, S. Y. Savrasov, and J.-M. Zuo, Phys. Rev. B 61, 2506 (2000). 